\title{
Placing Aboriginal kidney disease in context
}

\author{
Stephen P. McDonald PhD MBBS
}

See related research article by Jiang and colleagues on page 103 and at www.cmaj.ca/lookup/doi/10.1503/cmaj.130721

See also research article by Samuel and colleagues on page 110 and at www.cmaj.ca/lookup/doi/10.1503/cmaj.130776

I n a cohort study published in $C M A J$, Jiang

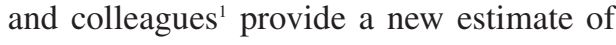
excess risk of end-stage renal disease among First Nations people in the province of Saskatchewan. Why is progression to end-stage renal disease more common among First Nations people than among non-First Nations people? The findings are not unique to Canada. Excess rates of the disease have been well documented in indigenous minority groups in the United States, ${ }^{2}$ Australia and New Zealand. ${ }^{3}$ The parallels are possibly greatest between Canada and Australia, both in the government, health care and geographic challenges and in the cultural and historical experiences of their indigenous people. In 2011, the age-standardized incidence of endstage renal disease among Aboriginal people in Canada was 41.9 per 100000 per year. ${ }^{4}$ When standardized to the same population base, Aboriginal Australians had a strikingly similar incidence rate of 41.6 per 100000 per year (unpublished data, Australia and New Zealand Dialysis and Transplant Registry). Although increased rates of diabetic nephropathy among indigenous people are widely known, rates of nondiabetic end-stage renal disease are also increased. ${ }^{5}$

A number of reasons have been advanced for these observations of increased incidence of chronic kidney disease among indigenous people, including genetic ("thrifty genotype") and environmental factors. One way to understand and reconcile these observations is through a multideterminant model, in which the occurrence of endstage renal disease is considered as the ultimate outcome of serial insults over decades. Among Australian Aborigines, increased rates of chronic kidney disease have been associated with low birth weight, which can lead to reduced nephron endowment and glomerulomegaly, ${ }^{6}$ lower renal volume, ${ }^{7}$ high frequency of bacterial infection and postinfectious renal damage related to glomerulonephritis, ${ }^{8}$ obesity-related hyperfiltration, and progressive vascular and diabetic damage leading to a reduced glomerular filtration rate. Individuals with chronic kidney disease likely have a number of these factors.
Jiang and colleagues provide a new perspective on this excess risk with their analysis of a province-wide dataset. "Lifetime risk" has intuitive appeal; it simplifies interpretation of longterm risk into a single number. The use of techniques to account for competing risk of censoring due to death is also important: use of traditional Kaplan-Meier methods will overestimate the cumulative incidence progressively with increasing age. The authors conclude that a component of the observed excess in incidence of end-stage renal disease among First Nations adults in their study can be attributed to the earlier age at onset of diabetes compared with non-First Nations adults. Nevertheless, at all ages of diabetes diagnosis, the incidence rates of end-stage renal disease and death were about three-fold greater among First Nations adults than among non-First Nations adults (although the excess risk of death lessened among those who were older when diabetes was diagnosed). ${ }^{1}$

The data from Jiang and colleagues' study also allow estimation of the cumulative risk of end-stage renal disease among First Nations people who have diabetes. For example, among First Nations people whose diabetes was diagnosed before 40 years of age, the 20-year cumulative incidence was about $10 \%$, rising sharply to about $15 \%$ after 25 years. ${ }^{1}$ Among First Nations people who were 40-60 years old when their diabetes was diagnosed, the 20-year cumulative incidence was a little less than $10 \%{ }^{1}$

\section{- KeY POINTS}

- First Nations people and indigenous peoples in the United States, New Zealand and Australia have similar rates and types of end-stage renal disease.

- End-stage renal disease in people with diabetes is the outcome of a series of processes: rates of end-stage disease are affected not just by the incidence of diabetes and progression to diabetic nephropathy, but also by competing mortality risks and the proportion of patients who start dialysis.

- The use of "lifetime risk" calculations provides a different perspective: the earlier age at onset of diabetes among First Nations adults means that a modest annual incidence of end-stage renal disease translates into a substantial cumulative lifetime risk. 
How do we place these long-term risks into context? A study based on data from the United States has estimated the lifetime risk of endstage renal disease to be $2.5 \%$ among white men. ${ }^{9}$ More recent Canadian work accounting for competing risks gave similar results (lifetime risk of $2.66 \%$ among men aged 40 without kidney disease).${ }^{10}$ Neither of these reports addressed the issue of risk among people with diabetes, nor are there data about lifetime risk of end-stage renal disease among First Nations people in general. In a study of a remote Australian Aboriginal community with particularly high rates of endstage renal disease, the overall lifetime risk was about $20 \%$, but it was as much as $40 \%$ among those with diabetes. ${ }^{11}$

There are a number of limitations to the interpretation of Jiang and colleagues' findings. The first is time: reaching the point of starting renal replacement therapy (dialysis or transplantation) is the culmination of a series of steps. Rates of end-stage renal disease depend on the rate of diabetic nephropathy among those with diabetes, the rate of progression to end-stage disease among those with diabetic nephropathy, competing mortality and, finally, the proportion of people with end-stage disease who start renal replacement therapy. The authors included people who received treatment over a 25 -year period, from 1980 to 2005. This period spans dramatic changes in management of diabetes. Treatments and treatment targets have evolved, and considerable evidence for interventions has emerged. It is feasible, therefore, that the cumulative risk of end-stage renal disease would be lower among people receiving a diabetes diagnosis today; paradoxically, the risk might be higher if the rates of competing vascular mortality were reduced further.

Second, treatment patterns for end-stage renal disease have changed over time. For example, renal replacement therapy has been offered more frequently to older patients in recent years. Increased availability of health care services for First Nations people in more remote communities may lead to higher rates of uptake of renal replacement therapy and therefore an even higher cumulative incidence of end-stage renal disease among First Nations people currently presenting with diabetes.

Finally, making inferences from small absolute numbers is challenging. The authors had information about very limited covariates (age at diagnosis, sex and ethnicity). Despite the accumulation of more than 90000 people in the cohort, there were only 200 cases of end-stage renal disease among the 8254 First Nations people. This will limit the power to observe effects of individual covariates as well as interactions.

Despite these limitations, Jiang and colleagues' findings should prompt researchers to consider studying strategies targeted at postponing the development of diabetes as a start to addressing rates of end-stage renal disease in indigenous populations. They also underline the importance of understanding epidemiologic principles of informative censoring and bias.

For clinicians and administrators, Jiang and colleagues' data indicate that the risk of renal disease increases progressively with increasing age. With the onset of diabetes at a younger age among First Nations people, end-stage renal disease is thus a common outcome. The challenge is not only to provide effective renal replacement therapy, but also to implement more effective primary prevention initiatives to delay the onset of diabetes and the progression of chronic kidney disease.

\section{References}

1. Jiang Y, Osgood N, Lim HJ, et al. Differential mortality and the excess burden of end-stage renal disease among First Nations people with diabetes mellitis: a competing-risks analysis. CMAJ 2014;186:103-9.

2. Narva AS. The spectrum of kidney disease in American Indians. Kidney Int Suppl 2003;63:S3-7.

3. McDonald SP, Russ GR. Current incidence, treatment patterns and outcome of end-stage renal disease among indigenous groups in Australia and New Zealand. Nephrology (Carlton) 2003;8:42-8.

4. End-stage renal disease among Aboriginal peoples in Canada: treatment and outcomes. Ottawa (ON): Canadian Institute for Health Information; 2013.

5. Dyck RF, Tan L. Non-diabetic end-stage renal disease among Saskatchewan Aboriginal people. Clin Invest Med 1998;21:33-8.

6. Hoy WE, Hughson MD, Singh GR, et al. Reduced nephron number and glomerulomegaly in Australian Aborigines: a group at high risk for renal disease and hypertension. Kidney Int 2006;70:104-10.

7. Spencer J, Wang Z, Hoy W. Low birth weight and reduced renal volume in Aboriginal children. Am J Kidney Dis 2001;37:915-20.

8. White AV, Hoy WE, McCredie D. Childhood post-streptococcal glomerulonephritis as a risk factor for chronic renal disease in later life. Med J Aust 2001;174:492-6.

9. Kiberd BA, Clase CM. Cumulative risk for developing end-stage renal disease in the US population. J Am Soc Nephrol 2002;13: 1635-44.

10. Turin TC, Tonelli M, Manns BJ, et al. Lifetime risk of ESRD. J Am Soc Nephrol 2012;23:1569-78.

11. Wang Z, Hoy WE. Diabetes and lifetime risk of ESRD in highrisk remote-dwelling Australian Aboriginal people: a 20-year cohort study. Am J Kidney Dis 2013;62:845-6.

Affiliations: Australia and New Zealand Dialysis and Transplant Registry, Renal Unit, Royal Adelaide Hospital; School of Medicine, University of Adelaide, Adelaide, Australia 